\title{
DETECTION OF SLOPE MOVEMENT BY COMPARING POINT CLOUDS CREATED BY SFM SOFTWARE
}

\author{
Kazuo Oda, Satoko Hattori, Toko Takayama
}

Asia Air Survey Co., Ltd. (kz.oda, stk.hattori, tk.takayama)@ajiko.co.jp

Commission V: WG V/3

KEY WORDS: Structure from Motion, ICP, point cloud, PCA, Slope Monitoring

\begin{abstract}
:
This paper proposes movement detection method between point clouds created by SFM software, without setting any onsite georeferenced points. SfM software, like Smart3DCaputure, PhotoScan, and Pix4D, are convenient for non-professional operator of photogrammetry, because these systems require simply specification of sequence of photos and output point clouds with colour index which corresponds to the colour of original image pixel where the point is projected. SfM software can execute aerial triangulation and create dense point clouds fully automatically. This is useful when monitoring motion of unstable slopes, or loos rocks in slopes along roads or railroads. Most of existing method, however, uses mesh-based DSM for comparing point clouds before/after movement and it cannot be applied in such cases that part of slopes forms overhangs. And in some cases movement is smaller than precision of ground control points and registering two point clouds with GCP is not appropriate. Change detection method in this paper adopts CCICP (Classification and Combined ICP) algorithm for registering point clouds before / after movement. The CCICP algorithm is a type of ICP (Iterative Closest Points) which minimizes point-to-plane, and point-to-point distances, simultaneously, and also reject incorrect correspondences based on point classification by PCA (Principle Component Analysis). Precision test shows that CCICP method can register two point clouds up to the 1 pixel size order in original images. Ground control points set in site are useful for initial setting of two point clouds. If there are no GCPs in site of slopes, initial setting is achieved by measuring feature points as ground control points in the point clouds before movement, and creating point clouds after movement with these ground control points. When the motion is rigid transformation, in case that a loose Rock is moving in slope, motion including rotation can be analysed by executing CCICP for a loose rock and background slope independently.
\end{abstract}

\section{INTRODUCTION}

Monitoring of loose rocks on slopes along the roads is quite important to keep traffic safety in Japan, where many roads are laid across mountainous area. This kind of monitoring is currently done by visual inspection. If inspectors cannot find the movement, the amount of movement is not measured and subcentimetre order movement might be missed.

SfM (Structure-from-Motion) software is now a powerful method for 3D reconstruction and point-cloud generation. SfM applications, such as Smart3DCaputure, PhotoScan, and Pix4D, are popular for professional and/or non-professional operator of photogrammetry. These systems directly process sequence of images and generate point clouds. Each point have colour index originated from colour of original image pixel. With photographing loose rock and surrounding environment, SfM software can calculate a point cloud which can realize quantitative estimation of rock movement.

Processing well-captured images results accurate point clouds. However, in many cases, the result is not constructed with correct scale or correct coordinates in reference coordinate system.

We have formerly proposed the evaluation method of correctness of shape of the point cloud created by SfM (Oda et al., 2015). The evaluation of this correctness uses the difference of point clouds with different source of images. It the shapes of the point clouds with different source of images is correct, two shapes of different source should be almost same. Comparison of the point has done after point cloud registration by iterativeclosest-point (ICP (Besl et al., 1992)), where transformation parameters (scale, rotation, and translation) are iteratively adjusted through minimization of sum of squares of distances. Distance in ICP should be determined as the distance between a point of one point cloud and its nearest face of the other point cloud. The distances of the two point clouds reflect the error of the two point clouds' shapes. This method can be applied for point clouds without correct scale. Some tests with test sample captured under indoor situation results that correctness of point clouds can be almost same as pixel resolution of original images (Oda et al., 2015)

This kind of registration can be applied to small movement detection of the slope, where some loose rock may fall from the slope. Detection of the change in two point clouds of different time can be executed, in common, by simply finding different part between two point clouds. In case that the change is very small, however, two point clouds should be measured precisely in common coordinates. In other word, changes smaller than precision of absolute coordinates of point clouds are imperceptible. If point-cloud generation uses ground control points (GCPs), movement smaller than precision of GCPs cannot be measured.

Another problem is that most of existing method uses meshbased DSM for comparing point clouds before/after movement and it cannot be applied in such cases that part of slopes forms overhangs. 
In this paper, the idea of evaluation of shape of point clouds is extended to motion analysis of loose rocks. The point cloud before movement is classified into two categorization: the rock part and the base part. Two parts are independently registered to point cloud after movement and transformation matrices are calculated. Motion of a loose rock is calculated as difference between two transformation matrices. This process can compensate the difference of coordinates between both point clouds.

This paper describes motion detection method. Point-cloud registration and shows an example of motion detection.

\section{METHOD OF MOTION DETECTION}

The motion detection method follows the procedure shown in Figure 1.

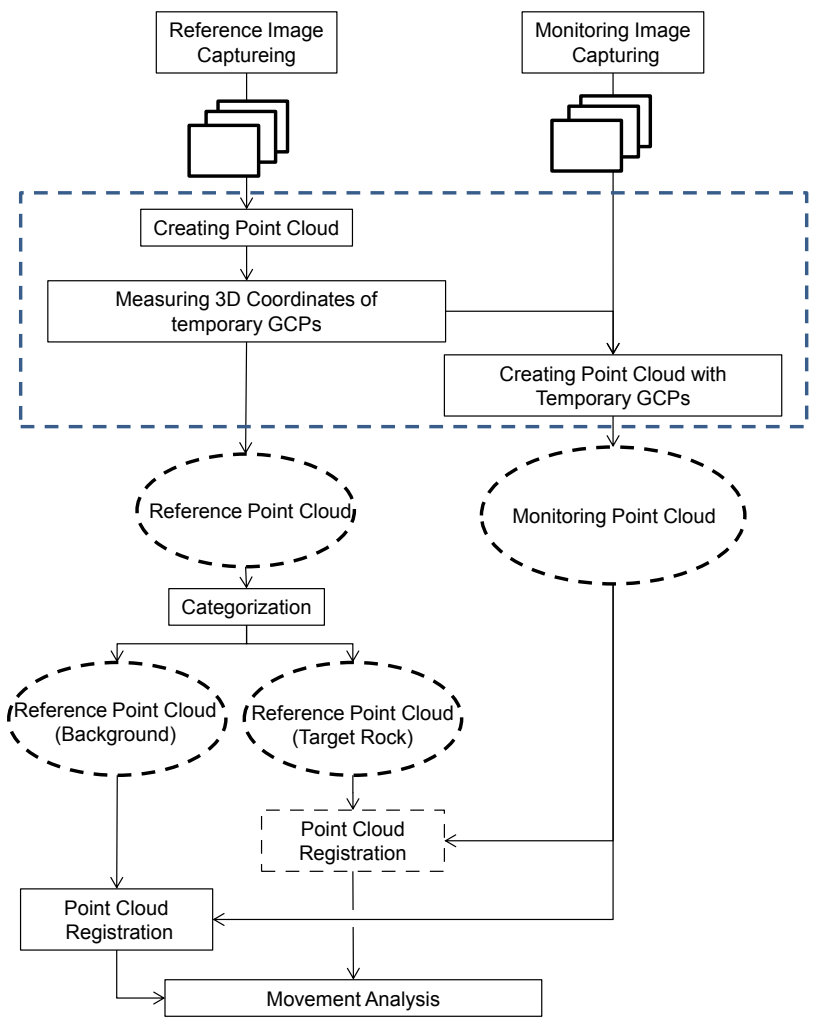

Figure 1. Procedure of the Motion Detection

\subsection{Capturing reference images and monitoring images}

Two image sets should be prepared: images before movement and currently monitoring images. Images before movement, or the oldest set of image set of the monitoring site, are regarded as reference image set. Each time of onsite monitoring, a set of images should be captured.

\subsection{Creating reference point clouds}

Reference point clouds of are generated by SfM software. Some eminent feature points which are not on a loose rock are chosen as temporary GCPs. Coordinates of these GCPs should be measured so as to be used as GCPs in point-cloud generation from sets of images after movement.

\subsection{Categorization}

The reference point cloud should be categorized into two categories: the target rock and the background. This categorization is done by manual interpretation so for, but operation has to be done just one time.

Categories can be recorded as an attribute of each point, for example, a classification code in a point data record of ASPRS LAS format.

\subsection{Creating the monitoring point cloud}

Point cloud is created from a set of monitoring images by SfM software. The monitoring point cloud should be in almost the same coordinate system as the reference point cloud. This can be attained by using the temporary GCPs in the point clouds in aerial triangulation. To avoid the deformation caused by systematic error of bundle adjustment in aerial triangulation, the errors of GCP coordinates should be large in bundle adjustment.

\subsection{Point-Cloud Registration}

Two set of registration are executed with ICP algorithm. One registration is between the target rock points in the reference point cloud and the monitoring point cloud, and the other is between the background points in the reference point cloud and the monitoring point cloud. Automatic registration process by ICP iteratively tries to minimize the distance of two point clouds. The source point cloud is moved to fit the target point cloud, by rigid transformation which is the combination of translation and rotation. Here the reference point cloud is regarded as the source point cloud and the monitoring point cloud is regarded as the target.

We adopt modified ICP algorithm called CCICP (Classification and Combined ICP) . The CCICP algorithm minimizes point-toplane, point-to-point distances, simultaneously, and also reject incorrect correspondences based on point classification by PCA (Principle Component Analysis) (Takai et al 2013). Before iterative process of ICP algorithm, each point is classified into linear points, planar points and scatter points according to the results of the PCA with neighbour points which are shown Figure 2 (Demanke et al 2011).

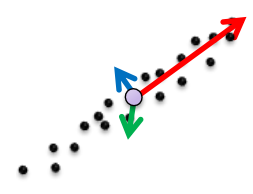

$\lambda_{1}>>\lambda_{2} \approx \lambda_{3}$

Linear Point

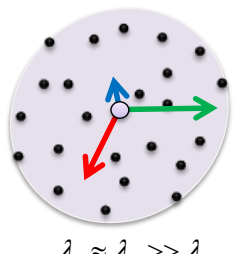

$\lambda_{1} \approx \lambda_{2}>>\lambda_{3}$

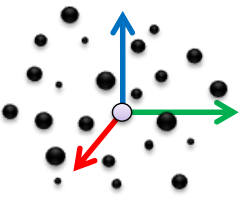

$\lambda_{1} \approx \lambda_{2} \approx \lambda_{3}$

Scatter Point
Figure 2. Point classification by PCA

Point-to-plane distance minimization is adopted to planar-planar correspondences and point-to-point distance minimization is adopted to the other correspondences. Point-to-plane and pointto-point distance minimization problem is done by the method of Low (Low, 2004). Definition of point-to-point distance (Dpt_pt) and point-to-plane distance (Dpt_pl) are following equation:

$$
\text { Dpt_pt }=\sqrt{\left|\left(T \cdot P_{S}-P_{t}\right)\right|^{2}}
$$




$$
\text { Dpt_pl }=\sqrt{\left|\left(T \cdot P_{s}-P_{t}\right) \cdot n_{t}\right|^{2}}
$$

where $\mathrm{T}$ is a transformation matrix in homogeneous coordinate system $\mathrm{P}_{\mathrm{s}}$ is a point in source point cloud, $\mathrm{P}_{\mathrm{t}}$ is the matching point in target point cloud, and $\mathrm{n}_{\mathrm{t}}$ is the normal vector of point $\mathrm{P}_{\mathrm{t}}$ calculated by PCA. The transformation matrix $\mathrm{T}$ can be described as following equation:

$$
\mathrm{T}=\left(\begin{array}{cccc}
a_{11} & a_{12} & a_{13} & \mathrm{t}_{\mathrm{x}} \\
a_{21} & a_{22} & a_{23} & \mathrm{t}_{\mathrm{y}} \\
a_{31} & a_{32} & a_{33} & \mathrm{t}_{\mathrm{z}} \\
0 & 0 & 0 & 1
\end{array}\right)
$$

where $a_{i j}$ are components of a rotation matrix and $\mathrm{t}_{\mathrm{x}}, \mathrm{t}_{\mathrm{y}}, \mathrm{t}_{\mathrm{z}}$ are translation. CCICP minimizes sum of square difference of corresponding coordinates of points (Takai et al, 2013).

The CCICP can fit the point cloud up to pixel order resolution of original images used in SfM process. The following sample shows it works for point cloud registration. Two point clouds in Figure 3 are a pair of point clouds of a slope failure created by Pix4DMapper from different set of images.

Aerial triangulation of the right side point cloud in Figure 3 had been used temporary GCPs measured in the left side point cloud in Figure 3. Both of point cloud should overlap each other, but there is slight gap between two point clouds as shown in Figure 4 (1). After automatic registration by CCICP, two point clouds had been strictly fitted.
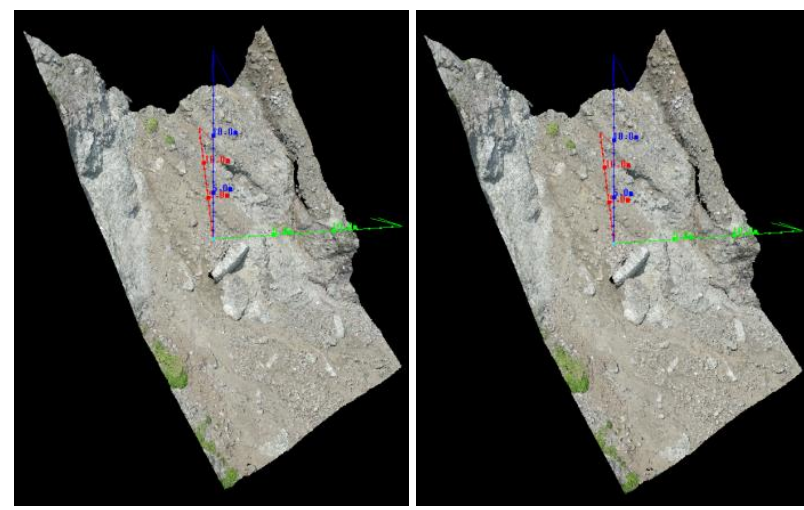

Figure 3. Point Clouds of a Slope Failure
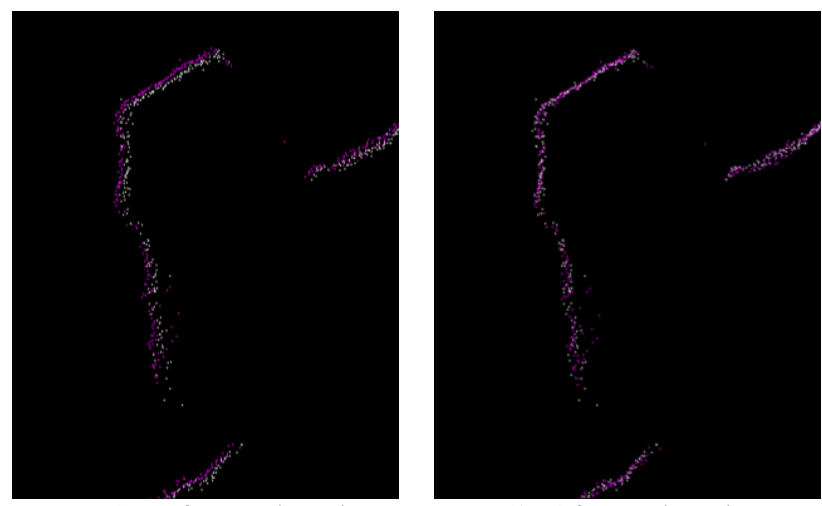

(1) Before registration

(2) After registration

\subsection{Movement analysis}

CCICP process calculates two transformation matrices. One is the matrix $T_{\text {rock }}$ which transforms the reference point clouds of the target rock to the monitoring point cloud, and the other is the matrix $T_{\text {back }}$ which transforms the reference point clouds of the background to the monitoring point cloud.

The movement matrix $T_{\text {move }}$ is calculated with following equation:

$$
T_{\text {move }}=T_{\text {back }}^{-1} \cdot T_{\text {rock }}
$$

\section{EXAMPLE OF MOVEMENT DETECTION}

A test had been executed to test this movement detection method. Two set of images had been captured where a large rock had moved manually. Figure 5 shows the two point clouds before and after movement of the large rock which is the centre of the figure. The rock had been manually moved. The first point cloud was used as the reference point cloud.

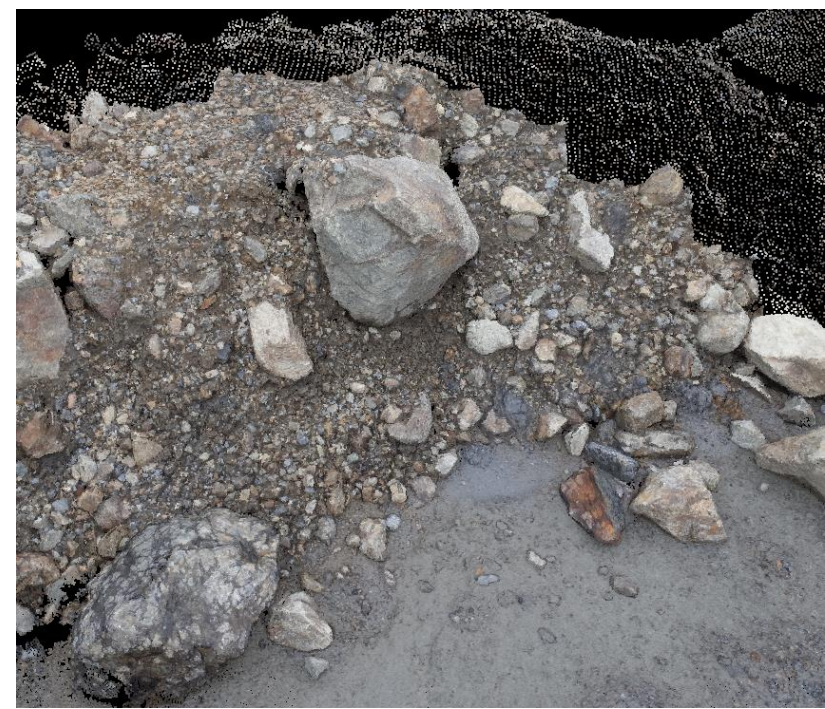

(1) A point cloud before movement

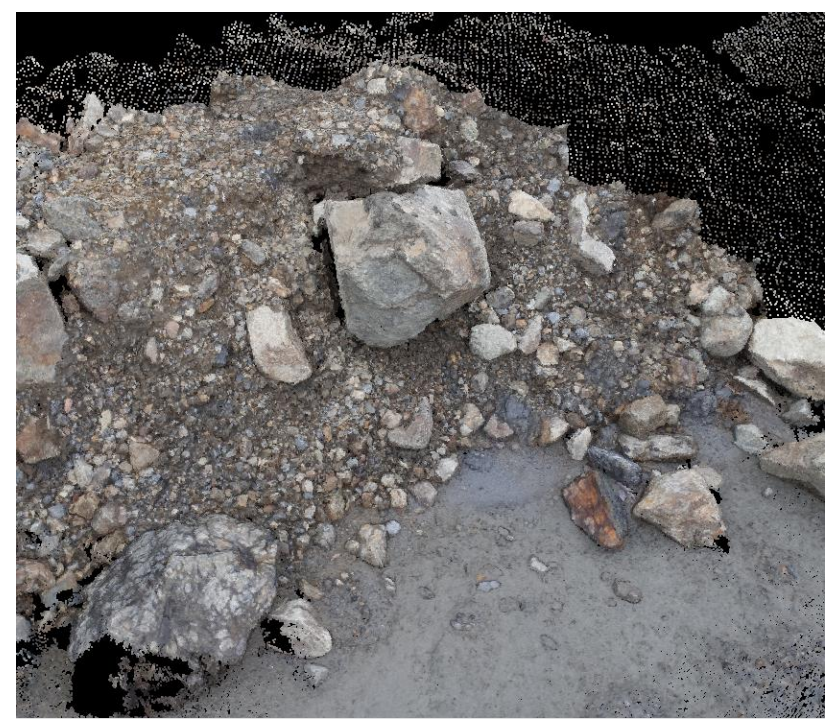

(2) A point cloud after movement

Figure 5. The effect of CCICP Registration 
The reference point cloud had been classified into the background part and the target rock part shown in Figure 6.

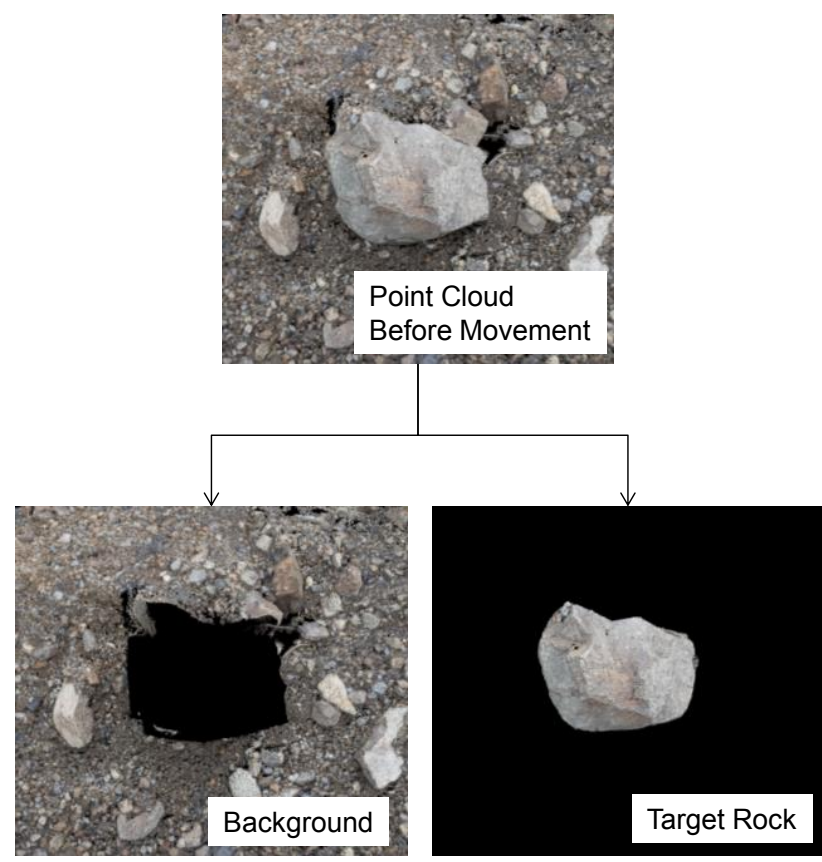

Figure 6. Categorization of the reference point cloud

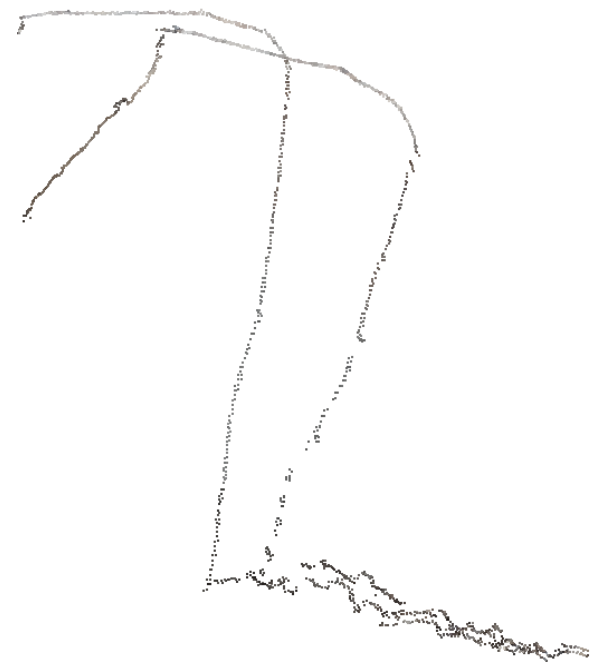

Figure 7. Profile of point clouds before / after movement

Figure 7 shows the calculated movement of the rock surface and Figure 8 shows movement vector of the rock surface. This method can calculate translation and rotation of the rock which includes overhanging surface.

\section{CONCLUSION AND FUTURE WORKS}

Movement analysis of loose rocks on a slope with point clouds generated by SfM has been proposed. Motion of a loose rock is calculated as difference between two transformation matrices: the matrix $T_{\text {rock }}$ which transforms the target rock part of the reference point clouds to the monitoring point cloud, and the other is the matrix $T_{\text {back }}$ which transforms the background of the reference point clouds to the monitoring point cloud. This process can compensate the difference of coordinates between both point clouds.

The extent of movement amount this method can manage depends on ICP process. Nonlinear optimization of ICP tends to fail when the movement is large. Further work for initial estimation of registration for the moving part will be the future works.

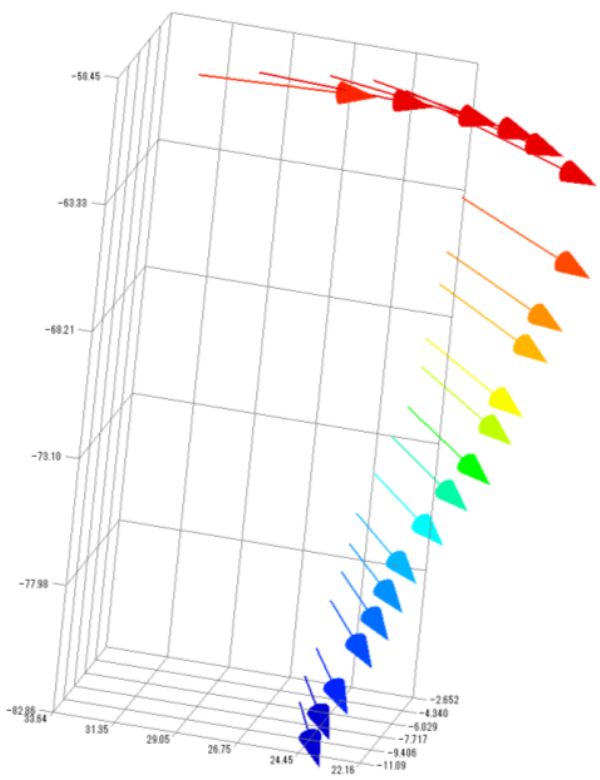

Figure 8. Calculated motion on the surface of the target rock

\section{ACKNOWLEDGEMENT}

We thank Prof. Satoshi Kanai and Assoc. Prof. Hiroaki Date, Hokkaido University, for help for CCICP programming and inspiring suggestion.

\section{REFERENCES}

Besl, Paul J.; N.D. McKay (1992). A Method for Registration of 3-D Shapes. IEEE Trans. on Pattern Analysis and Machine Intelligence (Los Alamitos, CA, USA: IEEE Computer Society) 14 (2): 239-256. doi:10.1109/34.121791.

K Oda, S Hattori, H Saeki, T Takayama, and R Homma (2015). Qualification of point clouds measured by SFM software, Int. Arch. Photogramm. Remote Sens. Spatial Inf. Sci., XL-4/W5, pp.125-130, 2015.

S. Takai, H. Date, S. Kanai, Y. Niina, K. Oda, and T. Ikeda(2013). Accurate registration of MMS point clouds of urban areas using trajectory, ISPRS Workshop Laser Scanning 2013, 277-282, Nov. 13th, 2013, Antalya, Turkey.

Demantke, J., Mallet, C., David. N., Vallet, B.(2011) Dimensionality based scale selection in 3D LiDAR Point Cloud. The International Archives of the Photogrammetry Remote Sensing and Spatial Information Sciences, 38 (Part 5/W12) (on CDROM). 